\title{
Simulation of Thermal Effects on the Flow Field in a Pilot-Scale Kiln
}

\author{
I. A. Sofia Larsson ${ }^{1}$ (D) - Anna-Lena Ljung ${ }^{1}$ - B. Daniel Marjavaara ${ }^{2}$
}

Received: 20 January 2021 / Accepted: 21 March 2021 / Published online: 20 April 2021

(C) The Author(s) 2021

\begin{abstract}
The flow field and coal combustion process in a pilot-scale iron ore pelletizing kiln is simulated using a computational fluid dynamics (CFD) model. The objective of the work is to investigate how the thermal effects from the flame affect the flow field. As expected, the combustion process with the resulting temperature rise and volume expansion leads to an increase of the velocity in the kiln. Apart from that, the overall flow field looks similar regardless of whether combustion is present or not. The flow field though affects the combustion process by controlling the mixing rates of fuel and air, governing the flame propagation. This shows the importance of correctly predicting the flow field in this type of kiln, with a large amount of process gas circulating, in order to optimize the combustion process. The results also justify the use of down-scaled, geometrically similar, water models to investigate kiln aerodynamics in general and mixing properties in particular. Even if the heat release from the flame is neglected, valuable conclusions regarding the flow field can still be drawn.
\end{abstract}

Keywords Rotary kiln $\cdot$ Computational fluid dynamics (CFD) $\cdot$ Coal combustion $\cdot$ Kiln aerodynamics

\section{Introduction}

One way to upgrade iron ore is to process it into pellets. This is done through a pelletizing process involving several steps including grinding, balling, and induration. In the GrateKiln induration process [1], the sintering is mainly taking place in a rotary kiln, where the pellets increase their strength and metallurgical properties. The kiln burner produces a flame that supplies the induration process with the necessary heat. Large amounts of process gas are supplied through channels with complex geometry, flowing through the kiln. The flame properties and characteristics are highly linked to the kiln aerodynamics due to the large amount of process gas supplied through the kiln hood. Since the flame is a diffusion flame, the mixing between the fuel (currently pulverized coal) and the combustion air is an important step of the combustion process and crucial for the combustion efficiency. A controlled and predictable flame reduces the fuel amount needed and hence the energy consumption

I. A. Sofia Larsson

sofia.larsson@1tu.se

1 Division of Fluid and Experimental Mechanics, Luleå University of Technology, SE-97187, Luleå, Sweden

2 LKAB, SE-98186, Kiruna, Sweden decreases, and the greenhouse gas emissions become lower. A long flame with an optimal temperature profile all the way through the kiln is also essential for a high product quality.

Due to the harsh environment with dust, high temperatures, and large mass flows of gas, measurements under operation are hard to perform; hence, simulation models can be a valuable tool for process understanding and control. Accurate numerical modelling relies heavily on experimental validation; therefore when striving toward valid models, a systematic study of simplified and down-scaled geometries provides an efficient way of moving forward step by step. A systematic study of the factors influencing the dynamics of the flow field in an iron ore grate-kiln pelletizing plant, by neglecting the combustion, has been performed in an ongoing project that has been running for several years [2-12]. Computational fluid dynamics (CFD) simulations of both simplified and full-scale geometries have been performed, as well as validating laser-based experiments in down-scaled, isothermal models. The next step is to increase the complexity of the model and include more physics to better describe the reality.

The objective of this work is to apply a coal combustion model in the CFD simulations of a pilot-scale kiln to enable an investigation of thermal effects from the flame on the surrounding flow field, such as volume expansion, density variations and buoyancy. The simulation results are partly compared to temperature measurements from the real pilot-scale kiln. 


\section{Method}

\subsection{Computational Model Overview}

The software Ansys CFX 2020 R2 is used for the simulations. It is an element-based finite volume method code where the governing equations are discretized and solved iteratively for each control volume defined by the computational grid. The governing equations are then integrated over each control volume such that the relevant quantities like mass, momentum, and energy are conserved. The result approximates the value of each variable at specific points throughout the domain, giving a full picture of the flow behavior [13].

A CFD model with coal combustion is employed for solving the flow field. To be able to identify the combustion effect on the flow field, simulations using a CFD model with only the hot gas flows are also performed. Important model simplifications include neglecting the pellet bed, the diameter expansion, and the rotation of the kiln to emphasize the focus on the flow field and combustion process. Figure 1 shows the pilot-scale kiln geometry with a closeup of the burner inlets. The kiln has a diameter of $0.65 \mathrm{~m}$ and is $12 \mathrm{~m}$ long. Figure 2 shows the real pilot-scale kiln. For details of the modelling equations, correlations, and constants, the interested reader is referred to the cited sources in the following sections.

\subsection{Gas Flow Field}

The turbulent flow field is solved by the three-dimensional, steady, Reynolds averaged Navier-Stokes (RANS) equations. These are closed by the two-equation $k-\varepsilon$ turbulence model with scalable wall functions, where two extra transport equations are solved - one for the turbulent kinetic energy and one for the dissipation.

The $k-\varepsilon$ model is commonly used when modelling industrial scale coal combustion in furnaces since it is

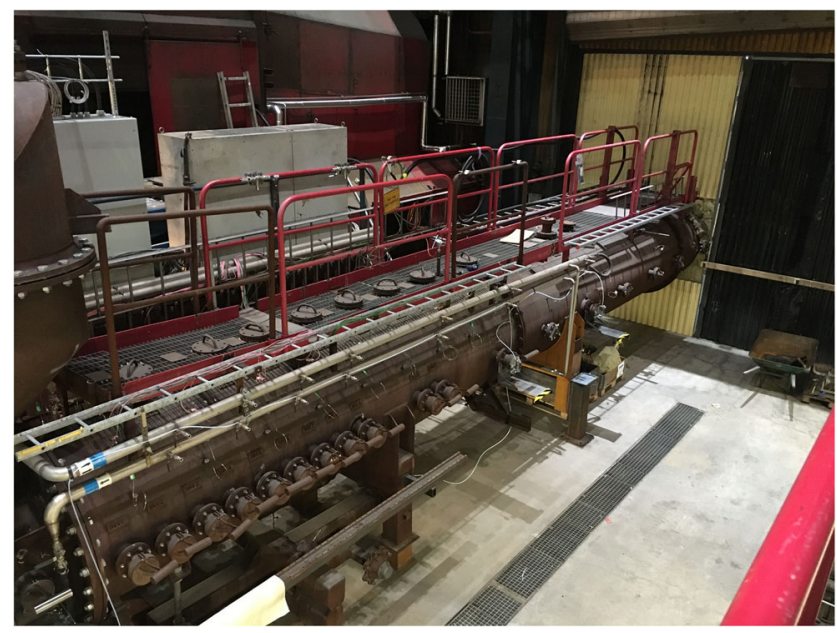

Fig. 2 Photo of the pilot-scale kiln modelled in the present work

robust and less computational expensive compared to more complex turbulence models. The drawbacks of the model are well known, especially its inability to accurately predict swirling flows with strong streamline curvature.

The energy equation, with the enthalpy as dependent variable, is solved for the thermal heat transfer and temperature field. In the combustion simulations, radiation plays a significant role in heat transfer between fluid and solids, and it is accounted for by adding a source term to the energy equation. This source term equals the net radiative heat flux in or out of the control volume and is obtained by solving a radiation transport equation. The choice of thermal radiation model affects both the quality of the solution and the computational time. In this work, the discrete transfer model is applied where the fluid domain is discretized into a radiation grid. From the center of each bounding surface, 32 rays are emitted, scattering radiation isotropically, and their paths are traced through the volume mesh. A sufficient number of rays are required for accurate calculation of thermal radiation in combustion processes. The discrete transfer model is combined with the gray
Fig. 1 Kiln geometry with a close-up of the burner inlets. The red dot in the middle corresponds to the coal and transport air inlet. The green surface shows the swirl air inlet and the blue surface represents the primary air inlet

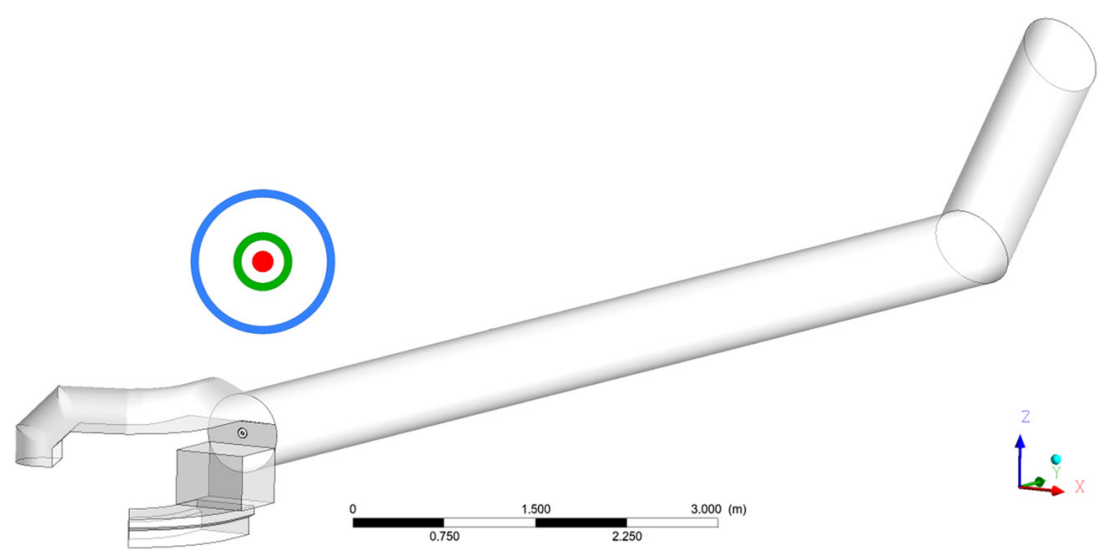


spectral model assuming that all radiation quantities are nearly uniform throughout the spectrum; consequently, the radiation intensity is the same for all frequencies [13].

\subsection{Combustion}

\subsubsection{Particle Transport}

The base of the combustion model employed consists of a hydrocarbon fuel model, which offers a simple method of creating a solid particulate combustion model starting from the ultimate and proximate analysis of the fuel. The pulverized coal is represented by a finite number of particles, tracked by a Lagrangian particle transport model. Each particle is tracked through the flow field and represents a sample of particles following an identical path. The particles are fully coupled to the gas flow, exchanging momentum with the continuous phase, implying that the particles affect the gas flow while the gas flow affects the particles simultaneously.

Particles dispersed in a continuous fluid are subjected to a number of forces. In this work, the drag force, the turbulent dispersion force, and the buoyancy force are accounted for. The drag force is calculated using the drag model of Schiller and Naumann [14] which was derived for flow past a single spherical particle.

Turbulent dispersion forces are important in turbulent flows with significant interphase drag. The result is additional dispersion of phases from high to low volume fraction regions due to turbulent fluctuations, caused by the combined action of turbulent eddies and interphase drag. The turbulent dispersion force is active in regions where the turbulent viscosity ratio exceeds a specified value; in this work, the default value of 5 is used [13].

The difference in density between the solid and fluid phases produces a buoyancy force which is accounted for in the simulation model with the specification of gravity. The buoyancy reference density is set as the average density of the continuous gas phase $\left(0.2 \mathrm{~kg} / \mathrm{m}^{3}\right)$.

The heat transfer between the continuous phase and the particles is modelled by the well tested correlation by Ranz and Marshall [15], which is based on boundary layer theory for steady flow past a spherical particle.

The composition of the coal particles will change during the combustion process. Since the particle emissivity depends on the particle composition, the variation with temperature is accounted for in the calculation of the particles effect in the radiation model.

As the particles are tracked through the flow domain, source terms are generated in addition to particle forces. The source terms account for heat release, consumption of $\mathrm{O}_{2}$, and production of $\mathrm{CO}_{2}$ and $\mathrm{H}_{2} \mathrm{O}$ due to the combustion reactions. The particle source terms acting on the fluid phase are proportional to the number of physical particles that a computational particle represents.

\subsubsection{Particle Reactions}

Three main reaction processes are modelled in coal combustion: devolatilization, char oxidation, and combustion of volatile matter. The initial drying of the coal particle is assumed to occur instantly as the particle is exposed to heat; hence, it is neglected in the model. The devolatilization is modelled using a single reaction model where raw coal is decomposed into char while volatile gases are released from the particle. The volatile yield is obtained by multiplying the proximate yield with a factor of 1.25 . During the volatilization, the total amount of material released is the actual volatile yield plus the moisture in the fuel. The relative amounts of carbon, hydrogen, and oxygen in the volatiles are calculated based on the ultimate analysis, which in turn determines the stoichiometric coefficients in the gas-phase combustion reactions involving the volatiles. The devolatilization rate is calculated by an Arrhenius type expression [13].

The remaining solids after the devolatilization consist of char and ash, where the char is assumed to be pure carbon. In the subsequent char oxidation model, a char particle is considered spherical and surrounded by a stagnant boundary layer through which oxygen must diffuse before it reacts with the char. The char reaction is hence determined by both the rate of oxygen diffusion to the surface and the oxidation reaction at the surface. The overall char reaction rate is controlled by the smallest of the two abovementioned rates [16].

The only solid remaining after the char oxidation is incombustible ash which can have a significant effect on the heat capacity, radiative heat transfer, and char surface reaction. To simplify the combustion model, the influence of ash is neglected.

The gas-phase combustion of the released volatiles is modelled by the single step reaction eddy dissipation model which is valid in turbulent flows where the chemical reaction rate is fast relative to the transport processes in the flow [17]. The reaction rate is assumed to be inversely proportional to a mixing time defined by the ratio of the turbulent kinetic energy, $k$, and the dissipation rate of $k, \varepsilon$.

\subsection{Simulation Settings}

The geometry is discretized into a grid consisting of 4.8 million tetrahedral and prism elements with 1.5 million nodes. Several grid densities were generated in a systematic way to check the sensitivity of the solution to spatial resolution. Regardless of grid size used, the main flow features in the kiln were the same. The details of the near 
wall flow were not of interest in this study; hence, wall functions were used. Close to the burner, the grid was refined to better capture the combustion kinetics. The computational grid can be seen in Fig. 3 .

The hot flow field without combustion was solved first and the solution was then used to initialize the combustion simulation. The coal size distribution is described by a Rosin-Rammler distribution with a mean diameter of $42 \mu \mathrm{m}$ and a spread constant of 1.008 . The coal properties can be seen in Table 1. One thousand representative particles uniformly injected, equally spaced over the coal inlet, were tracked in the combustion simulations.

Table 2 summarizes the boundary conditions used in all simulations (no coal particles were though injected in the hot gas flow simulations).

The solid surfaces are treated as opaque with an emissivity varying between 0.6 and 0.93 depending on type of wall. This implies that part of the radiation can travel through the surface while the other part is reflected and/or absorbed by the surface. An emissivity of 1 implies that the surface is treated as a black body.

The mass fraction of oxygen is set to $23.2 \%$ (corresponding to $21 \mathrm{vol} \%$ ) at all inlet and outlet boundaries.

The gas species that are solved for are the mass fractions of nitrogen $\left(\mathrm{N}_{2}\right)$, oxygen $\left(\mathrm{O}_{2}\right)$, water $\left(\mathrm{H}_{2} \mathrm{O}\right)$, and carbon dioxide $\left(\mathrm{CO}_{2}\right)$. Pollutants like $\mathrm{CO}$ and $\mathrm{NO}_{x}$ are neglected since they do not significantly affect the aerodynamics which is the focus of this work. Their reactions are usually modelled as a postprocessing step in the solver after the combustion of coal and air; hence, their one-way coupling has no effect on the combustion simulations and can therefore be neglected. In general, the combustion model was set up following the recommendations in the solver
Table 1 Coal analysis

Proximate analysis (as received basis, wt $\%$ )

Ash

13.3

Moisture

0.9

Fixed carbon

64.4

Volatiles

21.4

Ultimate analysis (as received basis, wt $\%$ )

Carbon

75.2

Hydrogen

3.8

Oxygen

5.101

Nitrogen

1.37

Chlorine

0

Sulfur

0.337

Heating value $29.726 \mathrm{MJ} / \mathrm{kg}$

documentation and default model constants are used in the equations for the devolatilization, char oxidation, and volatile combustion.

The high-resolution discretization scheme is used for the advection term, and this includes a blend factor that blends between first- and second-order accurate upwind schemes, ensuring a robust solution also in regions where the flow gradients change rapidly. The turbulence equations are discretized using a first-order upwind scheme.

The convergence criterion was root mean square (RMS) residuals below $10^{-6}$ for the hot gas flow simulations. The complex combustion simulations with strong coupling between the different phases and fast reactions, often resulting in instabilities, required a somewhat lower convergence target of RMS residuals below $10^{-5}$. Double precision

Fig. 3 Computational grid

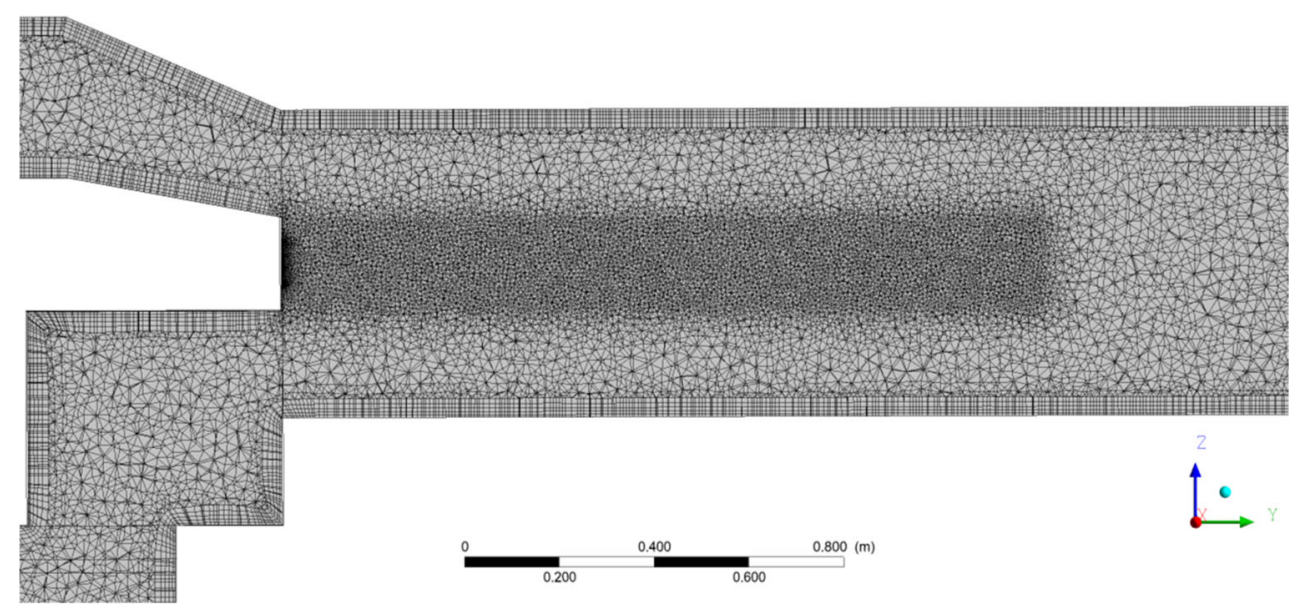


Table 2 Boundary conditions

\begin{tabular}{llll}
\hline & Gas mass flow rate $(\mathrm{kg} / \mathrm{h})$ & Coal mass flow rate $(\mathrm{kg} / \mathrm{h})$ & Temperature $\left[{ }^{\circ} \mathrm{C}\right]$ \\
\hline Coal inlet & 13 & 70 & 50 \\
Swirl air inlet & 41 with $30^{\circ}$ swirl & - & 50 \\
Primary air inlet & 35 & - & 50 \\
Upper secondary flow inlet & 1722 & - & 1075 \\
Lower secondary flow inlet & 1192 & - \\
Outlet & Average static pressure, relative pressure of 0 Pa & 1035 \\
Kiln wall & No slip, smooth wall with heat transfer coeff. of $2.27 \mathrm{~W} / \mathrm{m}^{2} \mathrm{~K}$ \\
Chimney & No slip, smooth wall with heat transfer coeff. of $20 \mathrm{~W} / \mathrm{m}^{2} \mathrm{~K}$ \\
Other walls & No slip, smooth wall, adiabatic \\
\hline
\end{tabular}

was used in all simulations. In order to allow the flow field to relax between the updates of the particle tracks and source terms, 50 iteration steps between successive calls to the particle solver were set in the combustion simulations. Monitor points were placed in the domain where the evolution of pressure, temperature, and velocity values could be followed to ensure a steady state solution. Mass imbalances of the final solutions were always well below $1 \%$.

\section{Results and Discussion}

The velocity in the YZ-center plane gives an overview of the flow field in the kiln, see Fig. 4. As expected, the combustion process with the resulting temperature rise and volume expansion leads to an increase of the velocity in the kiln. This is clearly seen in Fig. 4c showing the velocity difference between the cases. Naturally the velocity increase is largest close to the flame where the temperature is highest, but it can be seen throughout the entire kiln. Apart from that, the flow field looks similar regardless of whether combustion is present or not.

The velocity along the centerline (Fig. 5) also shows that the main difference between the cases is the velocity magnitude. The graphs show how the central burner jet emanates in the wake behind the so-called backplate separating the two secondary flow inlets. The velocity rapidly decreases initially until the flow from the lower secondary inlet reaches the centerline. The velocity then rapidly increases before reaching a local maximum. After that, the centerline velocity again decreases. The sudden peak around $\mathrm{y}=0.3 \mathrm{~m}$ in the combustion graph corresponds to the ignition point of the flame. The local minimum around $\mathrm{y}=1.5 \mathrm{~m}$ for the hot gas flow and $\mathrm{y}=2.2 \mathrm{~m}$ for the combustion case is an effect from the recirculation zone that develops below the jet emanating from the lower secondary flow inlet (see Fig. 4a and b). For the combusting case,
Fig. 4 Velocity contour in the YZ-center plane. a Hot gas flow. b Combustion. c Velocity difference between hot gas flow and combustion gas flow

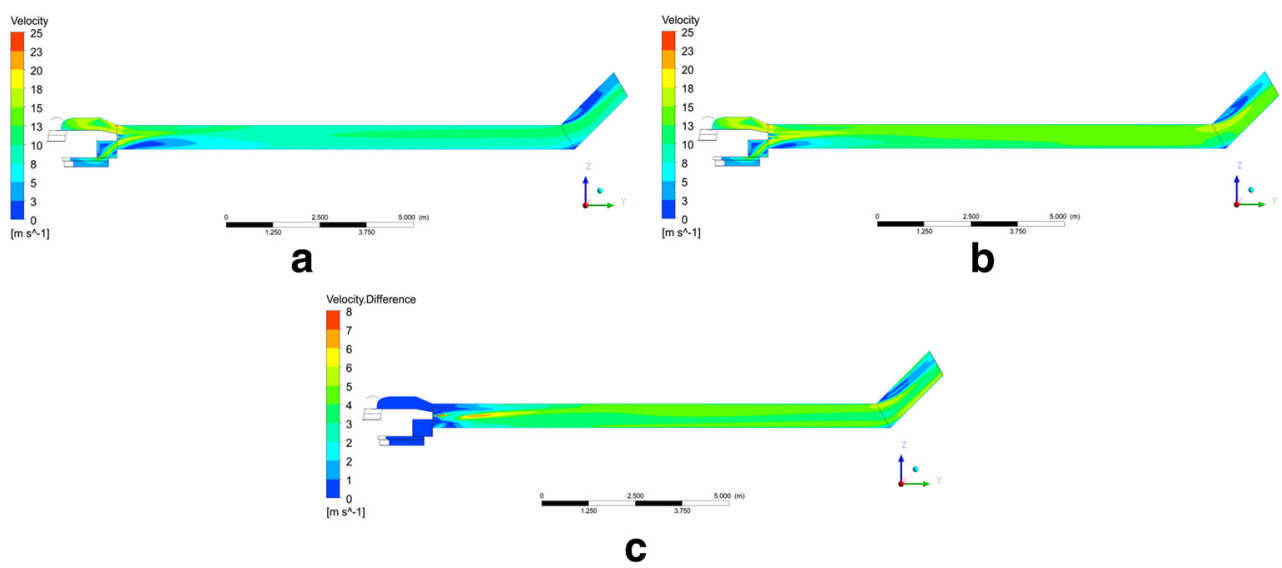


Fig. 5 Velocity along the centerline

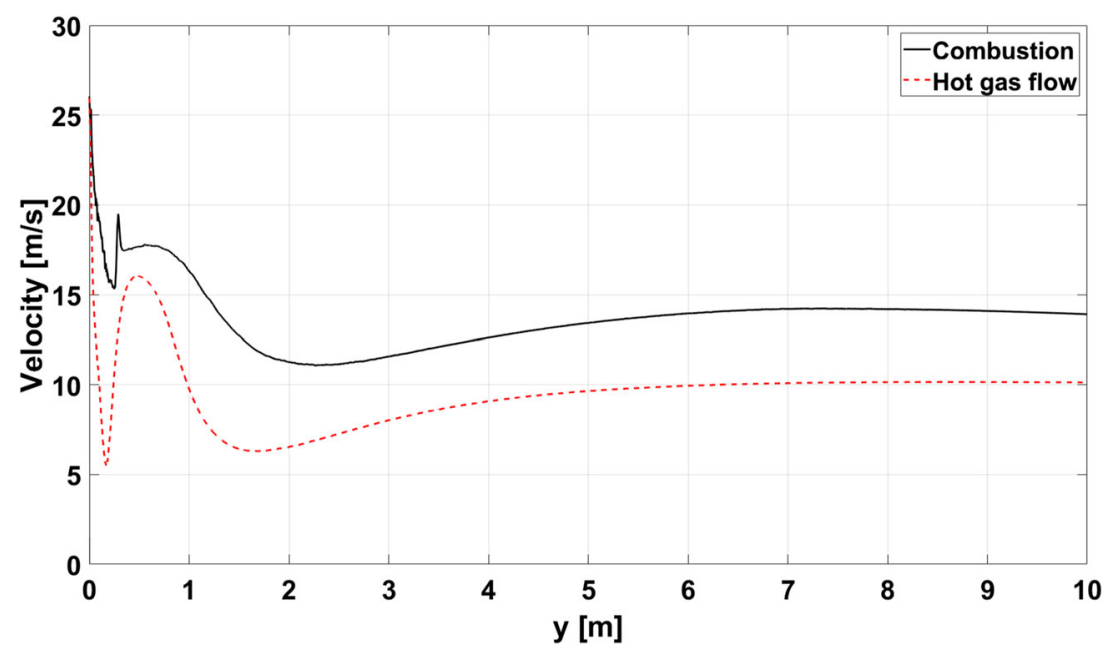

this recirculation zone is smaller; hence, its impact on the centerline velocity is less prominent which can be seen in the graph.

To get a better understanding of the complex flow field arising in the kiln due to the geometries of the secondary flow channels, velocity streamlines can be seen in Fig. 6. The streamlines are color coded depending on origin; streamlines emanating from the upper secondary inlet are red, streamlines from the lower secondary inlet are blue, and the streamlines originating from the burner inlets are all colored green. This makes it possible to get an idea of how the different flow streams interact with each other.

As can be seen, the velocity streamlines are far from straight and well-ordered, instead they are twisting and turning due to the flow phenomena, like detachment and recirculation, occurring in the kiln as a result of the complex geometries of the secondary flow inlet channels.
By following the evolution of the velocity in the XZplane downstream the kiln, it is again obvious that no significant differences, apart from the velocity magnitude, can be seen between the hot gas flow and combustion case, see Fig. 7. The vortices occur in the same places and the flow develops in a similar manner. Figure 8 shows the average velocity in the XZ-planes downstream the kiln. After the ignition of the flame and the start of the combustion process, the average velocity in the XZ-plane increases about $30 \%$ compared to the hot gas flow.

A closer look at the influence on the secondary flow streams by the combustion process reveals that no substantial distortion occurs in the YZ-center plane, see Fig. 9a and $b$. The merging streams are though closer to the centerline when combustion is present, suppressing the recirculation zone that develops in the lower part of the kiln (the blue zone below the jet emanating from the lower secondary
Fig. 6 Velocity streamlines in the kiln. Red streamlines originate in the upper secondary flow inlet, blue from the lower secondary inlet, and green streamlines emanate from all burner inlets. a Hot gas flow. b Combustion
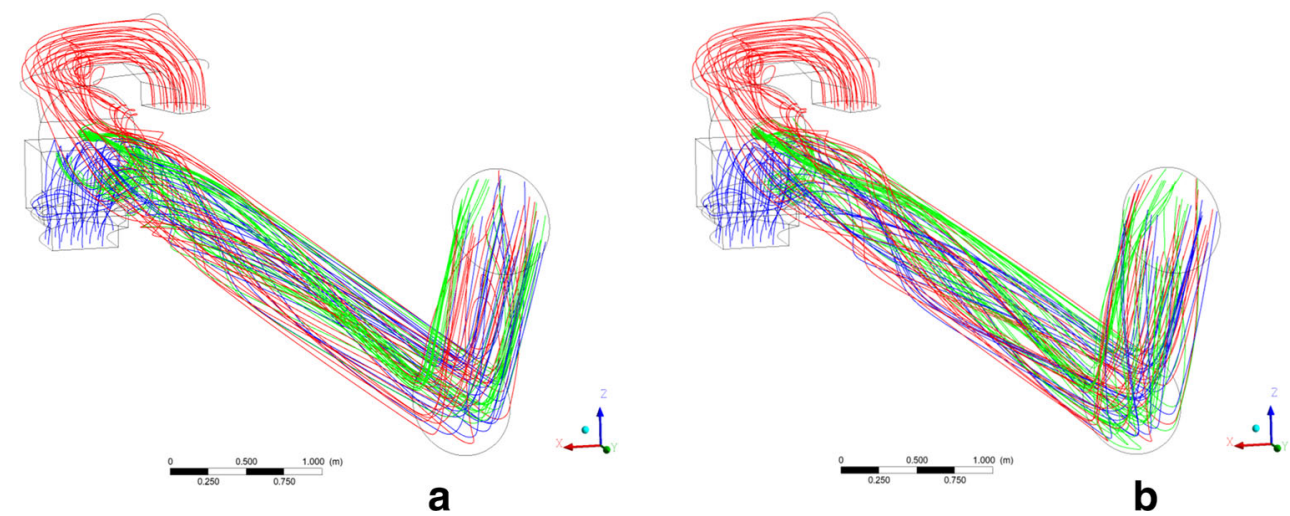
Fig. 7 Velocity contours with velocity vectors with tangential projection at four XZ-planes downstream the kiln. a Hot gas flow. b Combustion

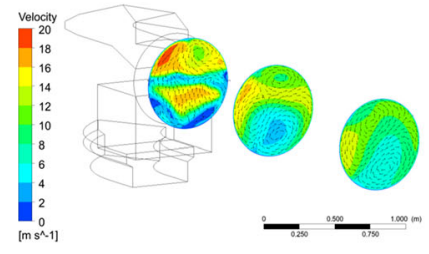

a

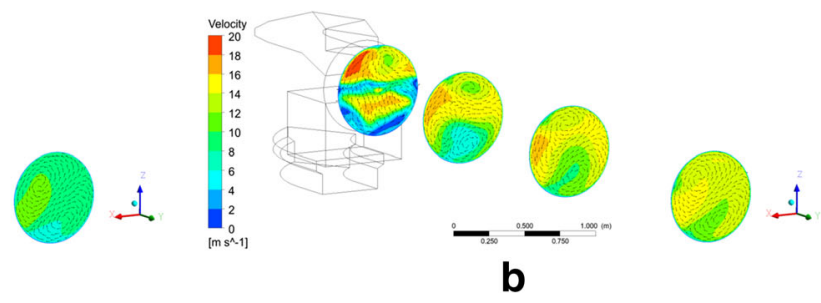

inlet). This is also clearly seen when looking at the XYcenter plane. It can also be seen that the flow is pushed toward the left side (corresponding to the upper side in Fig. 9c and d) and that a recirculation zone develops behind the backplate separating the two secondary inlets (the blue region near the burner inlet in Fig. 9c and d).

Since the focus in this work is how the combustion process affects the flow field, a deeper discussion about temperatures and species concentration fields etc. is left out. However, two contour plots (Fig. 10) showing the temperature in the YZ- and XY-center planes show how the flame ignites a short distance from the burner just outside the wake behind the backplate, and that the flame is confined in the vertical direction by the secondary flow streams, leading to an expansion in the lateral direction instead. The flame, following the flow field, is also pushed to the left side of the kiln, in accordance to Fig. 9. This is a result of the geometries of the secondary flow channels with complex cross-sections and bends in different directions, resulting in the secondary flow having a large impact on the flame propagation. Figure 11 shows an isocontour of $\mathrm{CO}_{2}$ mass fraction corresponding to a value of 0.15 , colored by temperature. This image gives an idea about the predicted flame shape and confirms the implications regarding the flame expansion from Fig. 10.

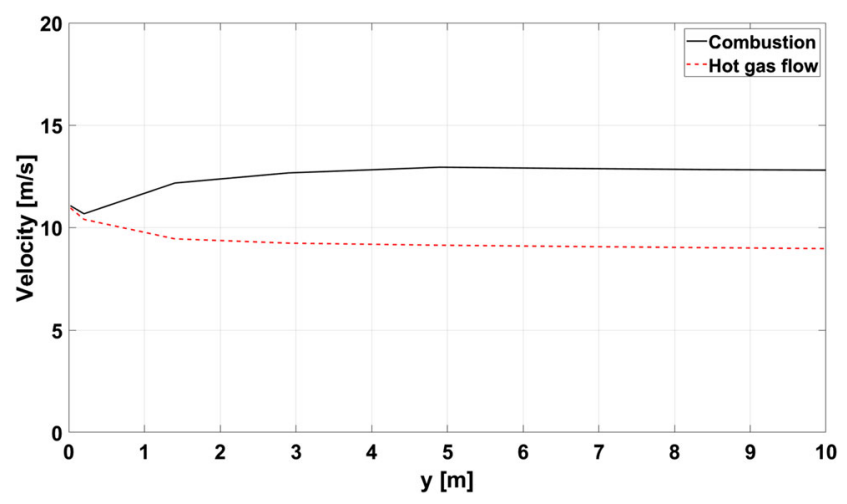

Fig. 8 Average velocity in XZ-planes downstream the kiln
Regarding the comparison to the real pilot-scale kiln, the only value applicable under similar operating conditions is the outlet temperature which was measured to $1320^{\circ} \mathrm{C}$ (a point measurement). In the combustion simulations, the kiln outlet temperature varied between 1318 and $1411^{\circ} \mathrm{C}$ over the area with an average value of $1393^{\circ} \mathrm{C}$, so the simulated temperature agrees well with the measured value. Several measurement campaigns have been performed in the experimental furnace and the present results regarding the direction of the flame qualitatively agrees with for example the work by Edland et al. [18]. It should though be noted that the coal properties and operating conditions differ, so a direct, quantitative comparison is not possible.

Several assumptions have been made regarding the combustion simulations and the reaction steps. This is a strong simplification from reality where several different species are involved in a large number of simultaneously occurring reactions. Nevertheless, it is clear from the present work that the heat release from the combustion process does not affect or change the overall flow field in any significant way. It is rather the opposite, the flow field affects the combustion process by controlling the mixing rates of fuel and air and governing the flame propagation. This shows the importance of correctly predicting the flow field in this type of kiln, with a large amount of process gas circulating, in order to optimize the combustion process. The results also justify the use of down-scaled, geometrically similar, water models to investigate kiln aerodynamics in general and mixing properties in particular. Even if the heat release from the flame is neglected, valuable conclusions regarding the flow field can still be drawn [19].

To better model the real process, more advanced submodels for both the combustion and the flow field is needed. The $k-\varepsilon$ turbulence model used in this work was chosen due to its robustness with less demands on computational power, especially in complicated coal combustion simulations, since the main focus was to disclose the combustion process' effect on the flow field. The choice of turbulence model is of utmost importance to accurately describe the complex flow field in this type of iron ore pelletizing rotary 

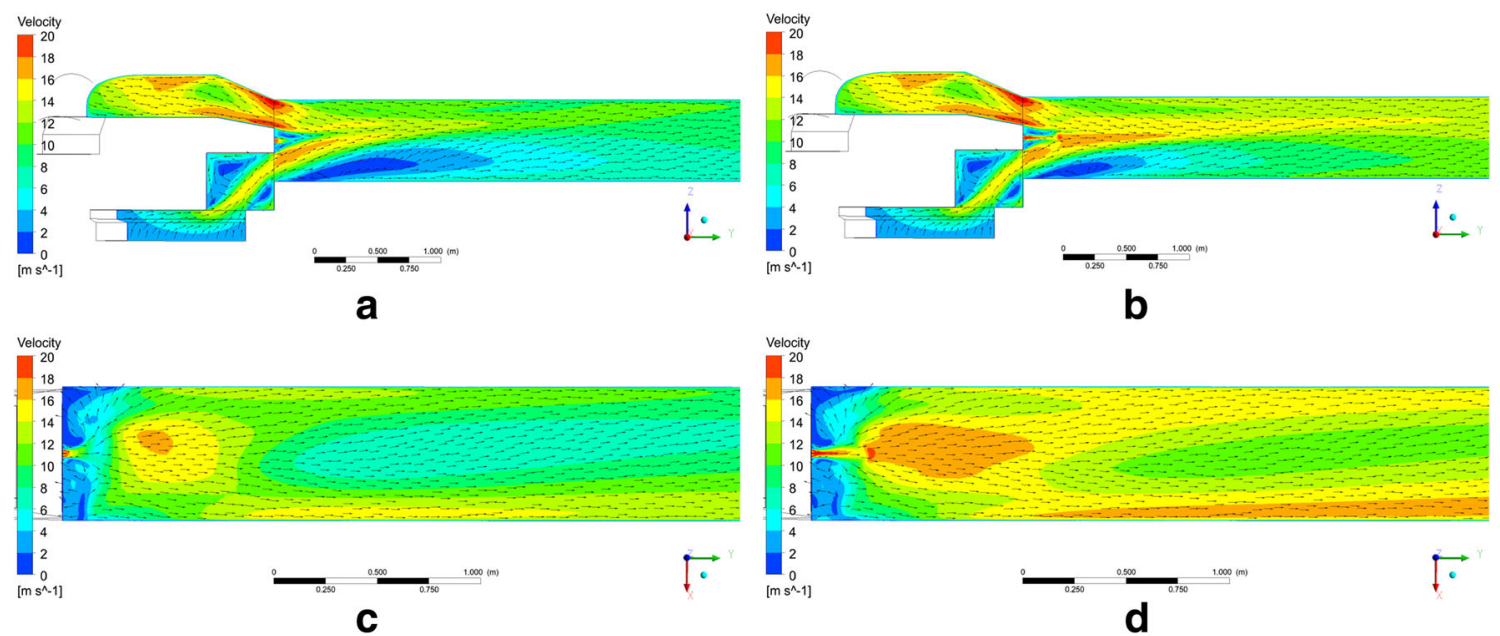

Fig. 9 Velocity vectors tangentially projected on the YZ-center plane on top, and the XY-center plane below. a Hot gas flow YZ-center plane. b Combustion YZ-center plane. c Hot gas flow XY-center plane. d Combustion XY-center plane

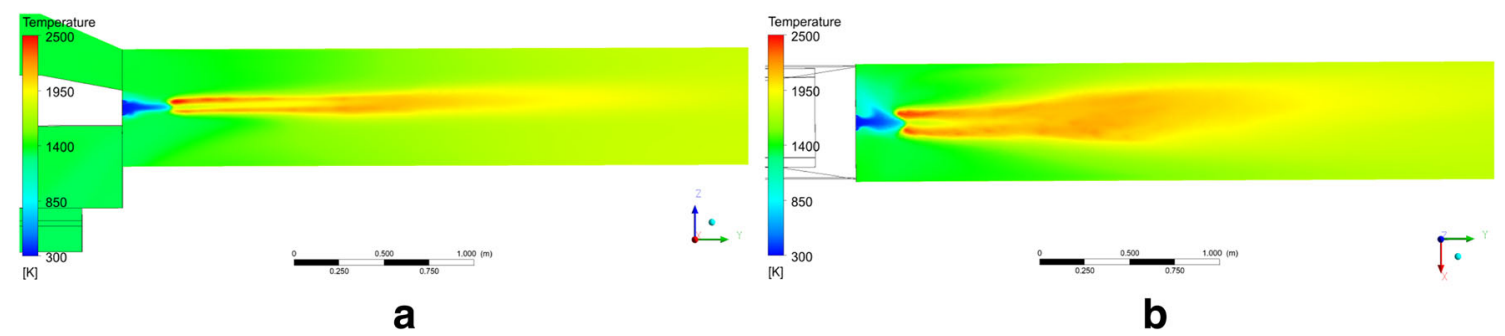

Fig. 10 Temperature contours in the YZ- and XY-center planes from the combustion simulation. a YZ-center plane. b XY-center plane

Fig. 11 Isocontour of $\mathrm{CO}_{2}$ mass fraction $=0.15$ colored by temperature

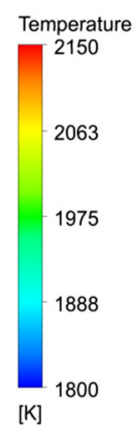

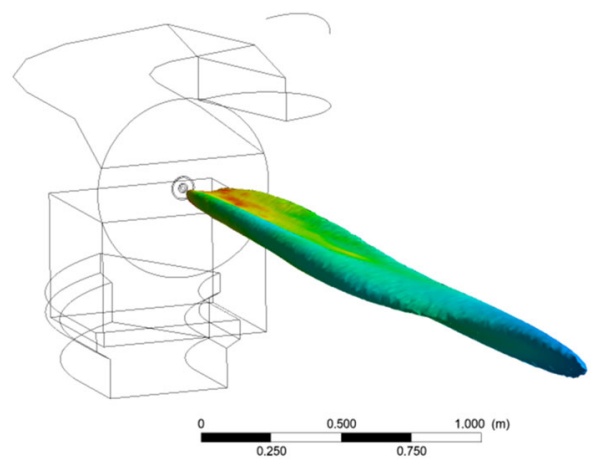

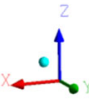


kiln, agreeing with previous conclusions in Larsson et al. [6, 8] and Granström [20].

\section{Conclusions}

The flow field and combustion process in a pilot-scale kiln was simulated in the commercial code ANSYS CFX. An initial coal combustion model was applied where the pulverized coal was treated as Lagrangian particles undergoing devolatilization and char oxidation with gasphase combustion of the released volatiles. The model can be used to capture general trends in the kiln and to get an overview of the process. It was concluded that the overall flow field in this particular kiln is not significantly affected by the heat release and gas volume expansion due to the flame. The flow field though affects the combustion process by controlling the mixing rates of fuel and air and governing the flame propagation. The pellet quality depends strongly on the temperature profile. A sound knowledge of the kiln aerodynamics and the resulting flow field is therefore necessary in order to optimize the combustion process to maximize the quality of the iron ore pellets produced.

Acknowledgements This work was carried out within the VINNOVA STRIM-project "New digital 3D model of the Grate-Kiln pelletizing process for reduced energy usage and emissions" (2017-02170). A special thanks also to Niclas Vestman and Julien Carmona for setting up the initial combustion model.

\section{Declarations}

Conflict of Interest The authors declare no competing interests.

Open Access This article is licensed under a Creative Commons Attribution 4.0 International License, which permits use, sharing, adaptation, distribution and reproduction in any medium or format, as long as you give appropriate credit to the original author(s) and the source, provide a link to the Creative Commons licence, and indicate if changes were made. The images or other third party material in this article are included in the article's Creative Commons licence, unless indicated otherwise in a credit line to the material. If material is not included in the article's Creative Commons licence and your intended use is not permitted by statutory regulation or exceeds the permitted use, you will need to obtain permission directly from the copyright holder. To view a copy of this licence, visit http://creativecommons. org/licenses/by/4.0/.

\section{References}

1. Stjernberg J, Isaksson O, Ion JC (2015) The grate-kiln induration machine - history, advantages, and drawbacks, and outline for the future. J Southern African Inst Mining Metall 115:137-144

2. Larsson IAS, Lindmark EM, Lundström TS, Nathan GJ (2011) Secondary flow in semi-circular ducts. J Fluids Eng 133(8):101206. https://doi.org/10.1115/1.4004991
3. Larsson IAS, Granström BR, lundström TS, Marjavaara BD (2012) PIV analysis of merging flow in a simplified model of a rotary kiln. Exp Fluids 53(2):545-560. https://doi.org/10.1007/ s00348-012-1309-1

4. Larsson IAS, Johansson SPA, Lundström TS, Marjavaara BD (2015a) PIV/PLIF experiments of jet mixing in a model of a rotary kiln. Exp Fluids 56(5):111(12). https://doi.org/10.1007/s00348015-1984-9

5. Larsson IAS, Lycksam H, Lundström TS, Marjavaara BD (2020) Experimental study of confined coaxial jets in a non-axisymmetric co-flow. Exp Fluids 61:256. https://doi.org/10.1007/s00348-02003094-3

6. Larsson IAS, Lundström TS, Marjavaara BD (2015b) Calculation of kiln aerodynamics with two RANS turbulence models and by DDES, Flow. Turbul Combust 94(4):859-878. https://doi.org/ 10.1007/s10494-015-9602-8

7. Larsson IAS, Lundström TS, Marjavaara BD (2015c) The flow field in a virtual model of a rotary kiln as a function of inlet geometry and momentum flux ratio. J Fluids Eng 137(10): 101102(11). https://doi.org/10.1115/1.4030536

8. Larsson IAS, Lundström TS, Marjavaara BD (2016) Simulation of the flow field in an iron ore pelletizing kiln. Minerals Metallurg Process 2016 33(3):144-148. https://doi.org/10.19150/mmp.6751

9. Burström P, Antos D, Lundström TS, Marjavaara BD (2015) A CFD-based evaluation of selective noncatalytic reduction of nitric oxide in iron ore grate-kiln plants. Prog Comput Fluid Dynam Int J 15(1):32-46. https://doi.org/10.1504/pcfd.2015.067327

10. Burström P, Lundstrym TS, Marjavaara BD, Töyrä S (2010) CFDModelling of selective non-catalytic reduction of NOx in gratekiln plants. Prog Comput Fluid Dynam Int J 10(5/6):284-291. https://doi.org/10.1504/pcfd.2010.035361

11. Teng Z, Johansson SPA, Larsson IAS, Lundström TS, Marjavaara D (2016) CFD simulation of jet mixing with asymmetric coflows in a down-scaled rotary kiln model. In: Proceedings of the ASME 2016 International Mechanical Engineering Congress and Exposition, vol 7. https://doi.org/10.1115/IMECE2016-65637

12. Teng Z, Larsson IAS, Lundström TS, Marjavaara BD (2018) The effect of Reynolds number on jet in asymmetric co-flows: a CFD study. Int J Chem Eng https://doi.org/10.1155/2018/1572576

13. ANSYS Inc (2020) ANSYS CFX-Solver Theory Guide, Release 2020R2, ANSYS Inc. Canonsburg, PA, USA

14. Shiller L, Naumann A (1935) A drag coefficient correlation. Zeitschrift des Vereins Deutscher Ingenieure 77:318-320

15. Ranz WE, Marshall WR (1952) Evaporation from drops, parts I \& II. Chem Eng Progress 48(3):141-146;173-180

16. Field MA (1969) Rate of combustion of size-graded fractions of char from a low-rank coal between $1200^{\circ}$ and $2000^{\circ}$. British Coal Utilisation Research Association 13:237-252

17. Magnussen BF, Hjertager BH (1977) On mathematical models of turbulent combustion with special emphasis on soot formation and combustion. In: 16th Symposium (International) on Combustion, vol 16, pp 719-729. https://doi.org/10.1016/S0082-0784(77) 80366-4

18. Edland R, Normann F, Fredriksson C, Andersson K (2017) Implications of fuel choice and burner settings for combustion efficiency and NOx formation in PF-fired iron ore rotary kilns. Energy \& Fuels 31(3):3253-3261. https://doi.org/10.1021/acs. energyfuels.6b03205

19. Mullinger P, Jenkins B (2008) Industrial and process furnaces: principles, design and operation, 1st edn. Butterworth-Heinemann, Oxford

20. Granström R (2012) Modelling the aerodynamics of iron ore pelletizing kilns. Licentiate thesis, Luleå University of Technology

Publisher's Note Springer Nature remains neutral with regard to jurisdictional claims in published maps and institutional affiliations. 